doi.org/I0.51891/rease.v7i8.2230

\title{
A PSICOPEDAGOGIA NA EDUCAÇÃO INFANTIL
}

\author{
Danielle Odilon Rodrigues ${ }^{1}$ \\ Elza Amaral Carlos Neto Nishino ${ }^{2}$ \\ Flávia Carmelita Duarte Souza ${ }^{3}$ \\ Hingridy Karoliny de Queiroz Arruda ${ }^{4}$ \\ Jaqueline de Moura Santana ${ }^{5}$ \\ Suelen Barros Berté6
}

RESUMO: O objetivo principal deste artigo é refletir sobre a importância de uma brincadeira para o desenvolvimento das crianças e, principalmente, para sua alfabetização. Assim, foi realizada uma revisão da literatura sobre conceitos de aprendizagem e dificuldades de aprendizagem, destacando sua relação com o desenvolvimento psicomotor e, assim, abordando a prevenção de distúrbios disgnosias e dispraxias como forma de prevenção de dificuldades de aprendizagem. Apresentamos diversos autores que consideram as habilidades necessárias à aquisição da leitura, da escrita e da matemática, habilidades essas que podem ser estimuladas pelas brincadeiras tradicionais. $O$ brincar desenvolve habilidades psicomotoras, espirituais, emocionais e até cognitivas; faz parte do desenvolvimento geral da criança. Entendemos que a proposta aqui apresentada pode ser um ponto de partida para a intervenção institucional de professores que atuam na educação infantil, para que possam refletir sobre sua prática na escola.

Palavras-chaves: Brincadeiras. Aprendizagem. Educação Infantil. Alfabetização.

ABSTRACT: The main objective of this article is to reflect on the importance of playing for children's development and, mainly, for their literacy. Thus, a literature review was carried out on concepts of learning and learning difficulties, highlighting their relationship with psychomotor development and, thus, addressing the prevention of dysgnosia and dyspraxia disorders as a way to prevent learning difficulties. We present several authors

\footnotetext{
I Graduada em Pedagogia pela UNIC - Universidade de Cuiabá, Especialista em Educação Infantil e Alfabetização pela AVEC - Associação Varzeagrandense de Ensino e Cultura / FIVE - Faculdades Integradas de Várzea Grande.

2 Graduada em Pedagogia pela UNIRODON, Especialista em Educação Infantil pelo Instituto FIC Faculdades Integradas de Cuiabá, Especialista em EJA - Educação de Jovens e Adultos pela AVEC Associação Varzeagrandense de Ensino e Cultura / FIVE - Faculdades Integradas de Várzea Grande.

3 Graduada em Letras - Língua Portuguesa / Literatura pela UFMT - Universidade Federal de Mato Grosso, Graduada em Pedagogia pela Faculdade INVEST de Ciências e Tecnologia, Especialista em Educação Infantil e Séries Iniciais pela Faculdade INVEST de Ciências e Tecnologia.

${ }^{4}$ Graduação em Pedagogia pela Universidade Norte do Paraná - UNOPAR, Especialista em Educação pela Faculdade Afirmativo.

5 Graduada em Pedagogia pela Universidade Norte do Paraná - UNOPAR.

6 Graduada em Pedagogia pela UNIC - Universidade de Cuiabá, Especialista em Educação Infantil e Alfabetização pela FACUMINAS EAD - Faculdade de Minas Gerais.
} 
who consider the skills necessary for the acquisition of reading, writing and mathematics, skills that can be stimulated by traditional games. Playing develops psychomotor, spiritual, emotional and even cognitive skills; it is part of the child's overall development. We understand that the proposal presented here can be a starting point for the institutional intervention of teachers who work in early childhood education, so that they can reflect on their practice at school.

Keywords: Jokes. Learning. Child education. Literacy.

\section{INTRODUÇÃO}

A psicopedagogia é a área do conhecimento que lida diretamente com as dificuldades humanas relacionadas à aprendizagem, pois estuda como esse processo ocorre (SCOZ, et al. I99I). Geralmente, o psicopedagogo escolar é contatado quando surgem dificuldades e sua função é avaliar e desenvolver um plano de intervenção. No entanto, a Psicodegagia não se caracteriza apenas como um campo de ação quando o problema ou dificuldade já existe, mas também por ser capaz de atuar de forma preventiva, evitando que isso aconteça. É com esse propósito que a Psicopedagogia Institucional se propõe. Nas escolas, os psicopedagogos atuam na dinâmica das instituições de ensino e capacitam os professores, orientando e auxiliando na organização das atividades e, portanto, no processo de aprendizagem de todos os alunos (MOOJEN, I990).

Nesta perspectiva de prevenção, entende-se que, graças aos diversos jogos tradicionais, podem ser evitadas certas dificuldades de aprendizagem relacionadas com a leitura, a escrita e a matemática que afetam diretamente o desenvolvimento psicomotor das crianças. O que ocorre é que, na maioria das vezes, as professoras de educação infantil não têm uma relação clara entre determinadas atividades recreativas e o futuro aprendizado dessas habilidades. Portanto, o objetivo deste estudo é mostrar que por meio de algumas brincadeiras comuns no cotidiano das crianças, podemos nos beneficiar com o desenvolvimento da leitura, da escrita e da matemática.

\section{DESENVOLVIMENTO}

2.I Aprendizagem e dificuldade de aprendizagem

É possível conceituar a aprendizagem a partir de diferentes perspectivas. Em uma perspectiva neurocientífica, a aprendizagem "é um processo complexo e dinâmico, que resulta em modificações estruturais e funcionais permanentes no sistema nervoso central" 
(OHLWEILER, 2006). Atos perceptivos e motores são elaborados pelo córtex cerebral, gerando aquelas modificações que, em última análise, originam a cognição (OHLWEILER, 2006).

Papalia \& Feldman (2013) defendem a ideia de que a aprendizagem acontece devido à plasticidade do cérebro, ou seja, a capacidade de se adaptar/modelar às mudanças no ambiente. Para Rotta (2006), a plasticidade cerebral é responsável tanto pela reorganização do sistema nervoso central após alguma lesão, como também é responsável pelo processo de desenvolvimento normal, pois está relacionada à flexibilidade que o cérebro tem de se modificar, à capacidade de aprender a partir da exposição a estímulos novos, ou seja, à cognição. De acordo com autora acima citada, as funções que envolvem a cognição como as gnosias (funções superiores ligadas ao conhecimento), praxias (capacidade de realizar um ato motor voluntariamente) e a linguagem são a expressão da plasticidade cerebral.

Para Henri Wallon, a ideia de aprendizagem está centrada na inter-relação dos domínios funcionais (afetividade, ato motor, ato mental), onde "a cada estágio, um dos domínios predomina e o exercício e amadurecimento de um, interfere no amadurecimento dos outros" (FREITAS \& MENEZES, 2012). Esse desenvolvimento se caracteriza como um processo dialético, e essa inter-relação acontece desde o nascimento. O bebê ao nascer é puro instinto, age impulsivamente para saciar suas necessidades; cabe ao meio (geralmente à mãe), interpretar seus gestos; gradativamente, o bebê vai percebendo e utilizando esses gestos de forma voluntária, iniciando o processo de comunicação e aquisição da linguagem, o início da vida psíquica.

A aprendizagem se dá nessa interação com o meio, que ora acontece por meio do aspecto motor, desenvolvimento e exploração corporal, através das experiências de interação e constituição da personalidade. A defasagem em um desses domínios interfere no desenvolvimento dos demais. Do ponto de vista neurológico, Rotta (2006) comenta que inteligência e praxia se constituem como "uma situação de causa e efeito, uma vez que não só a inteligência estrutura as praxias, como ela é um instrumento da inteligência”.

A Psicopedagogia vê a atenção aos aspectos neuropsicomotores como essencial para o processo de aprendizagem. A aprendizagem é um processo, no qual existem muitos aspectos envolvidos, como a atenção, memória, percepção, e também habilidades 
psicomotoras, como o equilíbrio, a manutenção da postura, motricidade ampla e fina, coordenação viso-manual, noção de ritmo e outros mais. Ao atendermos uma criança que apresenta dificuldades de escrita, os aspectos avaliados são seu desenvolvimento psicomotor, suas habilidades motoras. E, muitas vezes, o plano de intervenção tem como um de seus objetivos desenvolver as habilidades motoras que estão relacionadas aos movimentos que a criança precisa dominar com destreza para poder evoluir em seu grafismo. Para Corso (2007) "o desenvolvimento psicomotor está imbricado em todos os processos de aprendizagem".

As noções da educação psicomotora se aproximam dessa proposta, ratificando que a atenção ao desenvolvimento da criança desde o nascimento está diretamente implicada com seu processo de alfabetização e conhecimento matemático. Vayer (1982) define a educação psicomotora como sendo uma educação global, pois associa os potenciais intelectuais, afetivos, sociais, motores e psicomotores da criança, dando-lhe segurança, equilíbrio e organizando corretamente as suas relações nos diferentes espaços em que tem de atuar.

A educação psicomotora atua como intervenção preventiva, pois oportuniza que a criança se desenvolva melhor em seu ambiente (OLIVEIRA, 200I). Do ponto de vista psicopedagógico, é possível entender a atenção ao desenvolvimento psicomotor como prevenção às dispraxias e disgnosias.

Como dispraxias entende-se as "alterações no desenvolvimento do gesto, que é realizado em relação ao próprio corpo ou ao mundo dos objetos, relacionados a uma intenção" (ROTTA, 2006). Essas alterações podem se apresentar de diferentes formas, como por exemplo: a) dificuldade em usar adequadamente o lápis ou tesoura; b) realização de atividades de forma desastrada, derrubando o material, pois, geralmente, as crianças têm dificuldades em coordenar os movimentos necessários para executar a ação; c) dificuldades para colocar ou tirar as próprias roupas; d) lentidão ao participar de jogos e atividades próprios para a sua idade; e) dificuldade na montagem de quebra-cabeças; $f$ ) transtornos de conduta, dependendo do modo como essas alterações afetam sua noção de esquema corporal e suas relações com o espaço; $g$ ) distrações, problemas na fala e também na escrita (ROTTA, 2006). As dispraxias envolvem dificuldades da criança em relação a seu esquema corporal e 
noções de espaço e tempo, interferindo em sua autoconsciência e nas relações que estabelece com o mundo.

De acordo com Rotta (2006), as noções de esquema corporal envolvem funções como equilíbrio postural, lateralidade, freio inibitório, tônus muscular, e funções neuropsicológicas superiores (memória, atenção, percepção), as quais interagem entre si num determinado tempo e espaço com diferentes ritmos.

As disgnosias são alterações ou atrasos na integração das percepções. Essas percepções podem ser mais simples, como a auditiva, olfativa ou tátil, mas também podem ser mais complexas, como o esquema corporal e as noções de espaço e tempo. Existem diversos tipos de disgnosias. Dentre os principais sintomas/características pode-se citar: a) incapacidade de reconhecer objetos pelo tato, sem auxílio da visão; b) dificuldades ou atraso na capacidade de reconhecer sons não-verbais; c) dislalias que podem acarretar na troca de letras na idade escolar; d) dificuldade em identificar e reconhecer objetos, fisionomias, cores e espaços; e) dificuldade ou atraso na integração do esquema corporal; f) disfunção da noção de espaço, interferindo diretamente na noção corporal, temporal e no ritmo, geralmente levando à confusão direita-esquerda, em cima-embaixo, dentro-fora, perto-longe (OHLWEILER \& GUARDIOLA, 2006).

As disgnosias e dispraxias interferem no processo de leitura, escrita e também na matemática. Neste artigo, estamos abordando o processo preventivo de tais disfunções. “A organização das praxias e gnosias juntas são a base do desenvolvimento da inteligência, primeiro da inteligência sensoriomotora, depois da operatória e, por último, da inteligência abstrata e formal" (OHLWEILER \& GUARDIOLA, 2006). O professor deve conhecer como ocorre o desenvolvimento humano para poder auxiliar as crianças nesse processo.

\subsection{O papel do professor}

O professor deve ter clareza de que a criança é um ser integral e todos os aspectos precisam ser observados e desenvolvidos, pois estão interligados, bem como compreender a importância da brincadeira como um instrumento pedagógico. 
Desse modo, o brincar ajuda a promover a aprendizagem, pois "o comportamento de brincar é uma maneira útil de a criança adquirir habilidades desenvolvimentais - sociais, intelectuais, criativas e físicas" (MOYLES, 2006). O brincar pode trazer benefícios intelectuais para a criança, favorecer a habilidade da linguagem, incentivar o desenvolvimento cognitivo e a formação de conceitos. A brincadeira incentiva a criatividade e a imaginação através do jogo simbólico, e também estimula o desenvolvimento das habilidades motoras fina e ampla, uma vez que exercita/movimenta o corpo, desenvolvendo a coordenação motora.

Nesse sentido, o brincar deve ser considerado o principal instrumento pedagógico, em especial na educação infantil. Nessa faixa etária, a brincadeira é o meio mais significativo e interessante para as crianças de desenvolver objetivos pedagógicos, tendo em vista que as próprias habilidades de atenção, concentração e controle corporal, necessários para um ensino mais sistematizado, estão sendo desenvolvidas. Antes de aprender a ler e escrever, a criança precisa brincar.

Segundo Leal \& Luz (2006) existe uma grande contradição no processo educativo, pois a escola enfatiza o aprendizado de conteúdos específicos como a leitura e a escrita, mas não dá a devida importância às condições que afetam e influenciam diretamente a aquisição de tais habilidades.

O professor, ao organizar seu planejamento, deve conhecer o real potencial que as brincadeiras escolhidas por ele podem ter, objetivando contribuir de modo efetivo para a sua prática, quais aspectos estão implicados no processo de leitura, escrita e matemática, e como algumas brincadeiras contribuem para o seu desenvolvimento.

\subsection{O processo de leitura e escrita e brincadeiras}

Trevisan et al (2008) dizem que "o aprendizado da leitura depende de habilidades adequadas de processamento da fala [...]”. A nossa língua se caracteriza como um sistema alfabético, sendo necessário que a criança estabeleça a relação entre o grafema e o fonema durante o processo de alfabetização. Moojen (2009) utiliza o termo consciência fonológica para definir a capacidade de refletir e operar com os fonemas a partir da consciência de que 
as palavras são formadas por diferentes sons ou grupo de sons. Para a referida autora, existe uma relação de reciprocidade entre a consciência fonológica e a aquisição da leitura e escrita e, consequentemente, uma relação de hierarquia entre as habilidades envolvidas. Por exemplo, a identificação de um fonema no início das palavras é mais fácil do que no final; a segmentação parcial é mais fácil que a completa, que só é atingida com a aquisição e o exercício da leitura e escrita.

Para Lamônica (2008) a falha ou o atraso no desenvolvimento da linguagem interferem no aprendizado da leitura e da escrita porque a criança apresenta um vocabulário pobre e dificuldades para argumentar. É importante estimular a criança a dialogar, questionar, exercitando suas percepções sensoriais, de modo que tenha condições de reconhecer e discriminar as palavras e desenvolva a consciência fonológica, habilidade fundamental para o processo de aprendizagem da escrita (LAMÔNICA, 2008). Além dessas habilidades, a "atenção, discriminação, memória, análise e síntese" (Lamônica, 2008), também são responsáveis por esse processo. As alterações nessas habilidades faz com que as crianças tenham dificuldades em lidarem com os estímulos auditivos e visuais que são necessários para a aprendizagem da leitura e escrita.

De acordo com Capellini \& Souza (2008) para estarem aptas a escrever, as crianças precisam desenvolver várias habilidades, como o equilíbrio corporal, manipular objetos dentro da mão com destreza, bem como apreender e soltar objetos voluntariamente, ou seja, ter desenvolvido sua coordenação motora fina.

Scoz et al (1990) apontam outra situação importante. Esses autores afirmam que, quando crianças têm dificuldade de postura e de controlar seus movimentos, acabam tendo dificuldades de atenção e tais características podem ser encontradas em crianças que apresentam disgrafia, pois apresentam dificuldade em relação à organização espacial, não respeitando os limites das linhas e parágrafos.

É possível dizer que as habilidades a serem desenvolvidas são: a relação entra as letras e os sons; a posição de cada letra; sua localização no espaço; a direção da escrita. Obviamente, existem outras habilidades envolvidas, são suficientes para termos a noção de que o processo de leitura e escrita englobam habilidades bastante amplas como o equilíbrio corporal, noções de espaço e tempo e a acuidade visual e auditiva. Mesmo tratando-se de habilidades 
complexas, elas podem ser naturalmente vivenciadas e desenvolvidas pelas crianças quando se permite essa possibilidade através do brincar.

\subsection{Os princípios da matemática e brincadeiras que os favorecem}

Assim como na leitura e na escrita, as habilidades na área da matemática precisam que outras habilidades prévias sejam desenvolvidas. A criança ao chegar na educação infantil, traz consigo conhecimentos informais acerca do processo de contagem e que serão a base para a aprendizagem formal. O professor, deve permitir diferentes experiências para que o aluno seja desafiado e elabore seus conceitos.

Segundo Corso (2008), quando uma criança apresenta alguma dificuldade na área da matemática, é possível identificar como sintomas: as dificuldades na organização da memória de trabalho, certa lentidão na consolidação dos princípios de contagem, dificuldades em realizar os procedimentos de cálculo e nos processos executivos para a ativação de estratégias de recuperação e, ainda, dificuldades relacionadas à recuperação de fatos da memória de longo prazo.

De acordo com Bastos (2006), existem alguns sintomas que podem ser mais frequentemente detectados, como, por exemplo, escrita invertida de números, dificuldades para efetuar contas simples e reconhecer os sinais das operações, dificuldade de memorizar fatos numéricos, dificuldade em ordenar e organizar espacialmente os números nas operações de multiplicação e divisão. Nesse sentido, as diferentes experiências que podem ser estimuladas e proporcionadas na educação infantil, podem auxiliar as crianças a desenvolver essas habilidades.

É preciso proporcionar à criança experiências no âmbito do concreto, através da manipulação de materiais e da experiência com o próprio corpo (consciência do corpo no espaço). Bastos (2006) afirma que, por meio das experiências do dia a dia, devemos estimular a percepção de figuras e formas, a observação de seus detalhes, semelhanças, diferenças através de imagens e objetos e, a partir disso, trabalhar com os números e letras.

O professor deve ter clareza de que sua atuação, antes de chegar ao ensino de conceitos matemáticos específicos como os procedimentos de operações aritméticas, por 
exemplo, deve estar pautada na exploração de objetos, na observação do ambiente, na proposição de atividades lúdicas. Entre as habilidades que o professor pode estimular, estão os 'princípios de contagem' e 'senso numérico', aspectos fundamentais para a aprendizagem da matemática. Quanto aos princípios de contagem, a criança os adquire ao longo do seu desenvolvimento e a partir das experiências e interações que estabelece com o meio.

De acordo com Gelman \& Gallitel apud Corso (2008), são cinco os princípios que devem ser desenvolvidos pelas crianças:

- Ordem constante - a ordem da contagem dos números é sempre constante, portanto digo I, 2, 3, 4, 5 e não I, 3, 8, 9;

- Correspondência um a um (termo a termo) - para cada objeto tenho um nome de número;

- Cardinalidade - o valor do último número contado na série representa a quantidade de itens da série;

- Abstração - objetos de qualquer tipo podem ser colecionados e contados;

- Irrelevância da ordem - os itens dentro de um determinado grupo podem ser contados em qualquer sequência.

É importante que o professor possibilite atividades que contribuam para o desenvolvimento dessas habilidades..

O senso numérico está igualmente implicado na aprendizagem da matemática. Corso \& Dorneles (20ı) definem o senso numérico como "a facilidade e flexibilidade das crianças com números e a compreensão do significado".

Para que esse senso seja desenvolvido, compete ao professor permitir atividades que possam exercitar essas habilidades.

\section{CONSIDERAÇÕES FINAIS}

As brincadeiras podem estimular o desenvolvimento psicomotor das crianças e, portanto, favorecer o processo de leitura, escrita e matemática. As intervenções podem utilizar as brincadeiras, uma vez que o brincar faz parte do mundo infantil. É a partir daí que a criança começa a interagir com o mundo e se vê como parte dele. 
A brincadeira desenvolve habilidades psicomotoras, espirituais, emocionais e até cognitivas. Pode-se dizer que brincar é o primeiro passo para o desenvolvimento geral de uma criança.

Neste artigo, focamo-nos apenas numa das potencialidades do brincar infantil, nomeadamente o seu significado direto para o desenvolvimento neuropsicomotor das crianças e a sua influência no desenvolvimento de competências relacionadas com a aprendizagem da leitura, da escrita e da matemática.

\section{REFERÊNCIAS}

I. Scoz BJL, Barone LMC, Campos MCM, Mendes MH. Psicopedagogia: contextualização, formação e atuação profissional. Porto Alegre: Artes Médicas; I991.

2. Moojen S. Abordagem psicopedagógica da aprendizagem. In: Scoz BJL, Rubinstein E, Rossa EMM, Barone LMC, orgs. Psicopedagogia: o caráter interdisciplinar na formação e atuação profissional. Porto Alegre: Artes Médicas; 1990.

3. Ohlweiler L. Transtornos de aprendizagem. In: Rotta NT, Ohlweiler L, Riesgo R. Transtornos da aprendizagem: abordagem neurobiológica e multidisciplinar. Porto Alegre: Artmed; 2006.

4. Papalia DE, Feldman RD. Desenvolvimento humano. 12를 ed. Porto Alegre: AMGH; 2013.

5. Rotta NT. Dificuldades para a aprendizagem. In: Rotta NT, Ohlweiler L, Riesgo R, eds.

Transtornos da aprendizagem: abordagem neurobiológica e multidisciplinar. Porto Alegre: Artmed; 2006.

6. Freitas CN, Menezes ECP. A teoria psicogenética de Henry Wallon e a prática pedagógica em educação especial. In: Costas FAT, org. Educação, educação especial e inclusão: fundamentos, contextos e práticas. Curitiba: Appris; 2012.

7. Rotta NT. Dispraxias. In: Rotta NT, Ohlweiler L, Riesgo R, eds. Transtornos da aprendizagem: abordagem neurobiológica e multidisciplinar. Porto Alegre: Artmed; 2006.

8. Corso HV. Dificuldades de aprendizagem e atrasos maturativos: atenção aos aspectos neuropsicomotores na avaliação e terapia psicopedagógicas. Rev Psicopedagogia. 2007;24(73):76-89

9. Vayer P. A criança diante do mundo. Trad. Pabst MA. Porto Alegre: Artes Médicas; 1982.

Io. Oliveira GC. Psicomotricidade: educação e reeducação num enfoque psicopedagógico. $5^{\underline{a}}$ ed. Petrópolis: Vozes; 200I.

II. Ohlweiler L, Guardiola A. Disgnosias. In: Rotta NT, Ohlweiler L, Riesgo R, eds. Transtornos da aprendizagem: abordagem neurobiológica e multidisciplinar. Porto Alegre: Artmed; 2006.

I2. Moyles JR. A excelência do brincar. Porto Alegre: Artmed; 2006. 
13. Leal G, Luz MM. A intervenção psicopedagógica em crianças pré-escolares através do jogo de faz-de-conta. [Monografia de Especialização]. Campinas: Pontíficia Universidade Católica de Campinas; 2006. Disponível em http://bibliotecadigital.puccampinas.edu.br. Acesso em 26/09/2021.

I4. Trevisan BT. et al. Avaliação do vocabulário receptivo em crianças do ensino fundamental: teste de vocabulário por imagens Peabody (TVIP). In: Sennyey AL, Capovilla FC, Montiel JM, eds. Transtornos de aprendizagem: da avaliação à reabilitação. São Paulo: Artes Médicas; 2008.

15. Moojen SMP. A escrita ortográfica na escola e na clínica: teoria, avaliação e tratamento. São Paulo: Casa do Psicólogo; 2009.

16. Lamônica DAC. Disortografia: desafios para o aprendizado da escrita. In: Sennyey AL, Capovilla FC, Montiel JM, eds. Transtornos de aprendizagem: da avaliação à reabilitação. São Paulo: Artes Médicas; 2008.

17. Capellini SA, Souza AV. Avaliação da função motora fina, sensorial e perceptiva em escolares com dislexia do desenvolvimento. In: Sennyey AL, Capovilla FC, Montiel JM, eds. Transtornos de aprendizagem: da avaliação à reabilitação. São Paulo: Artes Médicas; 2008.

I8. Scoz BJL, Rubinstein E, Rossa EMM, Barone LMC, Beatriz J. Psicopedagogia: o caráter interdisciplinar na formação e atuação profissional. Porto Alegre: Artes Médicas; 1990.

19. Corso LV. A busca de relações entre as dificuldades na leitura e na matemática: um estudo com alunos de $3^{\mathrm{a}}$ a $5^{\mathrm{a}}$ série do ensino fundamental [Tese Doutorado]. Porto Alegre: Faculdade de Educação, Universidade Federal do Rio Grande do Sul; 2008.

20. Bastos JA. Discalculia: transtorno específico da habilidade em matemática. In: Rotta NT, Ohlweiler L, Riesgo R, eds. Transtornos da aprendizagem: abordagem neurobiológica e multidisciplinar. Porto Alegre: Artmed; 2006.

21. Corso LV, Dorneles BV. Senso numérico e dificuldades de aprendizagem na matemática. Rev Psicopedagogia. 2010;27(83)298-309. 